\title{
Some Peculiarities of Proton Transport in Quasi-One-Dimensional Hydrogen-Bonded Chains
}

\author{
Natalie I. Pavlenko \\ Institute for Condensed Matter Physics \\ 1, I. Svientsitsky Str., UA-79011, Lviv, Ukraine
}

(June 27, 2021)

\begin{abstract}
The protonic conductivity in the hydrogen bonded chains is investigated theoretically in the framework of the two-stage transport model. The strong interactions with optical phonon stretching mode are considered. We obtain the transition from the insulator to the metal-type state from the temperature dependencies of the hopping conductivity and analyze the influence of the specific Grotthuss mechanism on the transition character. We investigate also the main peculiarities in the frequency dependencies of the band and hopping conductivity parts which appear due to above-mentioned two-stage process of the proton migration along the chain.
\end{abstract}

72.20.i,66.30.Dn,71.30.+h

Typeset using REVTEX 


\section{INTRODUCTION}

The investigations of transport phenomena in hydrogen-bonded materials attract much attention due to their fundamental role in a variety of systems which are important for industrial, biological and other applications. It is well known that electric currents in these compounds are carried out almost entirely by mobile protons. On the one hand, this refers to proteins and biomembranes with one-dimensional channels for proton transport (for instance, proton pathways in bacteriorhodopsin) in which the proton transfer is directly in-

volved in many biochemical processes1. Such a materials are well known as sufficiently well protonic conductors with the conductivity in chain direction which exceeds $10^{3}$ times the conductivity in perpendicular direction 2. On the other hand, superprotonic phases discovered in some H-bonded systems are characterized by drastic increase of the protonic conductivity up to the values about $0.1 \Omega^{-1} \cdot \mathrm{cm}^{-1}$. It is generally accepted 3 目 that the two-stage conduction mechanism is required to sustain the proton transport. The intrabond proton tunnelling along the hydrogen bridge is connected with the transfer of ionic positive and negative charged defects, whereas the intermolecular proton transfer due to the reorientations of molecular group with proton leads to the breaking of the hydrogen bond and creation of a new one between another pair of molecular complexes (so-called orientational Bjerrum defects). It should be noted that the formation of the hydrogen bridge induces the distortion of groups involved in hydrogen bonding towards the proton that results in the shortening of this bond $\mathrm{b}$. By this means the protonic polaron is localized between distorted ionic groups at sufficiently low temperatures, giving rise in particular to the dimerized lowtemperature phases of some superprotonic conductors]. As has been shown in Ref. 8, the small polaron is formed due to the strong coupling of proton with the optical stretching vibration modes of the molecular groups.

Since the transport process has a two-stage character, the problem of the analysis of each stage contribution to the total proton mobility is of particular interest as far as there exist a systems with different relative concentration of ionic and orientational defects. It should be 
pointed out that whereas in systems like $\mathrm{KH}_{2} \mathrm{PO}_{4}$ a rapid dielectric relaxation is attributed to an abundance of mobile ionic defects and the rotation is sluggish, in ice the orientational $\mathrm{D}$ and $\mathrm{L}$ faults dominate the dielectric relaxation due to their greater numbers that limits the conductivity in ice. Indeed, the latter fact should manifest itself in the peculiarities of measured conductivity, and careful theoretical investigation could provide a possibility to support existing conjectures concerning the transport mechanism.

We start in this work with a double-minimum model for the protonic potential, which is modified by strong interaction with lattice vibrations. Aside from the proton motion in a double-well potential within the bond, we assume the interbond proton hopping which makes possible migration of protonic polaron along the chain. We neglect here the interproton correlation effects that can be valid in the case of low proton concentration in chain. However, we take into account the possibility of proton exchange between our selected chain and surrounding.

We study in this work the influence of ionic group displacements on the proton subsystem behavior. It is worthy to note that since the proton contribution into the electric current dominates in considered here H-bonded materials, the interaction of proton subsystem with the lattice distortions may lead to localization of protons and the system would therefore be an insulator. However, as we show in this work, the temperature dependence of the conductivity $\sigma$ changes from that typical of an insulator (decrease of $\sigma$ upon lowering $T$ ) to that typical of a conductor (increase of $\sigma$ upon lowering $T$ ). This effect points out to the existence of the so-called "metal"-insulator transition which was observed in the electronic semiconductors 9 . Moreover, the proposed here theoretical approach enables us to analyze the influence of the specific transport mechanism on the transition character. We show that in our case the transition to the "metallic" state with temperature increase has the peculiar two-step character which attributed to the two-stage nature of the proton migration process. Besides the specific temperature dependence, the above-mentioned complex transport mechanism manifests itself in the frequency dependencies of $\sigma$. Although we neglect initially the correlations between protons, it appears from the dynamical conductivity behavior, that 
the proton motion in the chain is a strongly correlated process (the neighbouring protons tend to move in pairs along the chain). While the first step of our analysis consists in the quasi-one-dimensional chains study, we believe our results can also be relevant for other hydrogen-bonded materials.

\section{DESCRIPTION OF THE MODEL}

The object of our consideration is the infinite chain shown in Fig. 11(a). However, to avoid the geometric complexities introduced by the kinks in such a zig-zag chain, we consider in our model linear chain (see Fig. 1(b) where two neighboring chains are shown). The process of the proton transfer in the double-well H-bond potential is represented as the quantum tunnelling between two proton states with the intrabond transfer integral $\Omega_{T}$

$$
\Omega_{T} \sum_{l}\left(c_{l a}^{+} c_{l b}+c_{l b}^{+} c_{l a}\right)
$$

where $c_{l \nu}^{+}, c_{l \nu}$ denote proton creation and annihilation operators in the position $(l, \nu=a, b)$ of the chain. Besides that, we describe the interbond reorientational proton hopping in two-level approximation as the quantum tunnelling effect with the hopping amplitude $\Omega_{R}$

$$
\Omega_{R} \sum_{l}\left(c_{l+1, a}^{+} c_{l b}+c_{l b}^{+} c_{l+1, a}\right) .
$$

In this way within the framework of orientational-tunnelling model proposed in Ref. 10 the two-stage proton migration mechanism can be considered as the sequential migration of the ionic and orientational defects. The proton energy spectrum and dielectric properties of the finite H-bonded complexes were analyzed on the basis of exact diagonalization procedure 1 .

As far as such a double-well chain is just a structural component of the system we also admit a possibility of proton exchange between the chain and surroundings by considering the system thermodynamics in the framework of the grand canonical ensemble with inclusion of the proton chemical potential

$$
-\mu \sum_{l, \nu} n_{l \nu}
$$


which is to be determined at the given proton concentration in the chain from corresponding equation for the chemical potential.

Our main interest here is to analyze the influence of the longitudinal optical ionic group vibration modes on the proton subsystem transport properties. We consider the optical in-phase stretching vibrations of ionic groups in the chain which induce their displacements with respect to the surrounding chains as identified in Fig. 1(b) by dashed arrows. The interactions of protons with this optical mode cause the difference of the potential minima $(l, a)$ and $(l, b)$ depth within the bond

$$
\sum_{l, q} \tau_{l}(q)\left(n_{l a}-n_{l-1, b}\right)\left(b_{q}+b_{-q}^{+}\right) .
$$

Here $\tau_{l}(q)=g \sqrt{\hbar / 2 M N \omega(q)} \exp [i q l d]$ where $g$ is the corresponding coupling constant, $M$ is the effective ionic group mass, $N$ denotes the number of hydrogen bonds in chain and $d$ is the lattice parameter. The optical phonon branch creation and annihilation operators are denoted by $b_{q}^{+}$and $b_{q}$ respectively. Furthermore we assume the harmonic approximation for the lattice vibration energy

$$
\sum_{q} \hbar \omega(q) b_{q}^{+} b_{q}
$$

First of all let us consider the case of the isolated chain without a coupling to the phonon bath. Since the Hamiltonian (11)-(3i) can be exactly diagonalized, the proton energy spectrum

$$
\varepsilon_{\nu}(k)= \pm\left|t_{k}\right|, t_{k}=\Omega_{T}+\Omega_{R} \mathrm{e}^{-i k d}
$$

forms two energy bands with the bandwidth $\Delta \varepsilon=\Omega_{T}+\Omega_{R}-\left|\Omega_{T}-\Omega_{R}\right|$. The energy gap in this case is $\Delta_{a b}=2\left|\Omega_{T}-\Omega_{R}\right|$. Eliminating one of the elementary transport process by setting the hopping amplitude $\Omega_{T}=0$ or $\Omega_{R}=0$, we can see that both of the energy bands degenerate into the two energy levels and the quantum fluctuations between these two system states could be derived. It is clear that in the case when $\bar{n}=\frac{1}{N} \sum_{l \nu}\left\langle n_{l \nu}\right\rangle=1$ (one proton is averaged within the bond) the lower band is filled and the chemical potential $\mu$ is centered between bands - thus the material is in insulator state. However, for $\bar{n}=\frac{1}{2}$, as an 
example, only half of the lower band is filled and this corresponds to the case of protonic conductor that occurs for example in superionic phases of superprotonic crystals.

We will discuss afterwards the consequences of the proton-phonon coupling effect focusing on the analysis of the strong coupling regime. Since the low-order perturbation theory is insufficient in this case, we apply a canonical Lang-Firsov transformation

$$
U=\mathrm{e}^{i S}, \quad S=\sum_{l \nu} n_{l \nu} v_{l \nu}, \quad v_{l a}=-v_{l-1, b}=i \sum_{q} \frac{\tau_{l}(q)}{\hbar \omega(q)}\left(b_{q}-b_{-q}^{+}\right) .
$$

We present the transformed Hamiltonian

$$
\tilde{H}=\mathrm{e}^{i S} H \mathrm{e}^{-i S}=\tilde{H}_{0}+\tilde{H}_{t}
$$

where

$$
\begin{aligned}
\tilde{H}_{0} & =-\tilde{\mu} \sum_{l \nu} n_{l \nu}+E_{0} \sum_{l} n_{l a} n_{l-1, b}+\sum_{q} \hbar \omega(q) b_{q}^{+} b_{q} \\
\tilde{H}_{t} & =\Omega_{T} \sum_{l}\left(\Phi_{T}^{l} c_{l a}^{+} c_{l b}+\Phi_{T}^{l *} c_{l b}^{+} c_{l a}\right)+\Omega_{R} \sum_{l}\left(\Phi_{R}^{l} c_{l a}^{+} c_{l-1, b}+\Phi_{R}^{l *} c_{l-1, b}^{+} c_{l a}\right) .
\end{aligned}
$$

Here $\tilde{\mu}=\mu+E_{0}$ is the proton chemical potential which is renormalized due to the lattice polarization and the formation of the protonic polaron as the result. The notation $E_{0}=$ $\sum_{q}\left|\tau_{l}(q)\right|^{2} / \hbar \omega(q)=(\hbar g)^{2} / 2 M\left(\hbar \omega_{0}\right)^{2}$ denotes the binding energy of the small polaron which forms in the strong proton-phonon coupling regime and the phonon operators

$$
\Phi_{T}^{l}=\mathrm{e}^{-\sum_{q} \frac{\tau_{l}(q)+\tau_{l+1}(q)}{\hbar \omega(q)}\left(b_{q}-b_{-q}^{+}\right)}, \quad \Phi_{R}^{l}=\mathrm{e}^{-2 \sum_{q} \frac{\tau_{l}(q)}{\hbar \omega(q)}\left(b_{q}-b_{-q}^{+}\right)},
$$

give rise to the protonic polaron band narrowing factors with their thermal averages given by

$$
\left\langle\Phi_{T}^{l}\right\rangle=\mathrm{e}^{-\sum_{q}\left|\tau_{l}(q)\right|^{2} \operatorname{cth} \frac{1}{2} \beta \hbar \omega(q) /(\hbar \omega(q))^{2}}, \quad\left\langle\Phi_{R}^{l}\right\rangle=\mathrm{e}^{-2 \sum_{q}\left|\tau_{l}(q)\right|^{2} \operatorname{cth} \frac{1}{2} \beta \hbar \omega(q) /(\hbar \omega(q))^{2}}=\left\langle\Phi_{T}^{l}\right\rangle^{2} .
$$

Furthermore we consider the case of $\bar{n}=1$ with the filled lower proton band which corresponds to the insulator state of the system. 


\section{CURRENT OPERATOR AND OPTICAL CONDUCTIVITY}

To analyze the protonic conductivity along the chain direction, we should evaluate the following expression in the framework of the linear response Kubo theory 14

$$
\sigma(\omega, T)=\frac{1}{N d} \int_{0}^{\infty} d t \exp [i(\omega+i \varepsilon) t] \int_{0}^{\beta} d \lambda\langle J(t-i \hbar \lambda) J(0)\rangle,
$$

where the proton current operator $J=\frac{e}{i \hbar}[H, x]=J_{T}+J_{R}\left(x=\sum_{l \nu} n_{l \nu} r_{l \nu}\right.$ is the proton polarization operator and $r_{l \nu}$ is the proton coordinate in the $\nu$ th position of the $l$ th bond). After the transformation (7) the parts $J_{T}$ and $J_{R}$ which represent the intrabond and interbond proton transfer respectively, have the following form

$$
\begin{aligned}
& \tilde{J}_{T}=\frac{e \Omega_{T}}{i \hbar} R_{a b} \sum_{l}\left(\Phi_{T}^{l} c_{l a}^{+} c_{l b}-\Phi_{T}^{l *} c_{l b}^{+} c_{l a}\right), \\
& \tilde{J}_{R}=\frac{e \Omega_{R}}{i \hbar} R_{r} \sum_{l}\left(\Phi_{R}^{l *} c_{l-1, b}^{+} c_{l a}-\Phi_{R}^{l} c_{l a}^{+} c_{l-1, b}\right),
\end{aligned}
$$

where $R_{a b}$ is the separation between the potential wells within the H-bond and $R_{r}$ is the distance between the nearest proton positions on the neighbouring bonds adjacent to the ionic group. In this case the polaronic bandwidths $\Delta \varepsilon=\tilde{\Omega}_{T}+\tilde{\Omega}_{R}-\left|\tilde{\Omega}_{T}-\tilde{\Omega}_{R}\right|\left(\tilde{\Omega}_{T}=\Omega_{T}\left\langle\Phi_{T}^{l}\right\rangle\right.$, $\left.\tilde{\Omega}_{R}=\Omega_{R}\left\langle\Phi_{R}^{l}\right\rangle\right)$ narrow exponentially with temperature increase due to the proton-phonon scattering processes. Because of this, the polaronic bands degenerate practically to localized states and the proton migration proceeds as the hopping of the protonic polaron between localized positions in the lattice at high temperatures.

It is well known that the total polaronic conductivity can be represented as a sum of two parts11: $\sigma=\sigma_{c o h}+\sigma_{\text {hop }}$. The band conductivity $\sigma_{c o h}$ dominates at low temperatures, whereas the hopping term $\sigma_{\text {hop }}$ makes the main contribution at high temperatures, when the bandwidths are small and the particles become trapped at individual sites due to phonon interactions. We consider first the protonic low-temperature band conductivity. Since the scattering caused by phonon interactions can be neglected in this case, the proton transport is assumed to be the motion in the thermally averaged phonon field. Then evaluation of the correlation functions in (12) using the Wick's theorem yields the following expression for the real part of $\sigma_{c o h}$ 


$$
\sigma_{c o h}=\sigma_{c o h}^{0}+\sigma_{c o h}^{a b}
$$

where the Drude-type term can be written as

$$
\sigma_{c o h}^{0}=-\pi \frac{e^{2} \beta}{4 \hbar^{2} N d} \sum_{k, \nu} \frac{\left(\tilde{\Omega}_{k} \tilde{t}_{k}^{*}-\tilde{\Omega}_{k}^{*} \tilde{t}_{k}\right)^{2}}{\left|\tilde{t}_{k}\right|^{2}}\left\langle\tilde{n}_{k \nu}\right\rangle\left(1-\left\langle\tilde{n}_{k \nu}\right\rangle\right) \delta(\omega)
$$

with $\tilde{\Omega}_{k}=\tilde{\Omega}_{T} R_{a b}-\tilde{\Omega}_{R} R_{r} \mathrm{e}^{-i k d}, \tilde{t}_{k}=\tilde{\Omega}_{T}+\tilde{\Omega}_{R} \mathrm{e}^{-i k d}$, and $\sigma_{c o h}^{a b}$ is given by

$$
\sigma_{c o h}^{a b}=\frac{e^{2}}{\hbar d} \frac{\operatorname{sh} \beta \hbar \omega / 2}{(\hbar \omega)^{2}} \frac{1}{\operatorname{ch} \beta \tilde{\mu}+\operatorname{ch} \beta \hbar \omega / 2} \frac{\left[\left(\tilde{\Omega}_{T}^{2}-\tilde{\Omega}_{R}^{2}\right)\left(R_{a b}+R_{r}\right)+(\hbar \omega / 2)^{2}\left(R_{a b}-R_{r}\right)\right]^{2}}{\sqrt{\left.\left(2 \tilde{\Omega}_{T} \tilde{\Omega}_{R}\right)^{2}-\left[(\hbar \omega / 2)^{2}-\left(\tilde{\Omega}_{T}+\tilde{\Omega}_{R}\right)^{2}\right)\right]^{2}}}
$$

Here the notations $\left\langle\tilde{n}_{k \nu}\right\rangle=\left[1+\exp \left(\beta\left( \pm\left|\tilde{t}_{k}\right|-\tilde{\mu}\right)\right)\right]^{-1}$ denote thermally averaged Fermi distributed polaronic band occupancies. We see that the first term $\sigma_{c o h}^{0}$ corresponds to the intraband transfer processes giving rise to the absorption peak at $\omega=0$, whereas $\sigma_{c o h}^{a b}$ is associated with the interband transfer processes and leads to appearance of the van-Hovetype singularities in the absorption at $\hbar \omega= \pm\left(\tilde{\Omega}_{T}+\tilde{\Omega}_{R}\right)$ and $\hbar \omega= \pm\left|\tilde{\Omega}_{T}-\tilde{\Omega}_{R}\right|$.

\section{THE PHONON-ACTIVATED PROTON TRANSPORT}

Furthermore, we will focus on the analysis of the polaronic transport which corresponds to phonon-activated hopping, because of the dominant contribution of this part to the total protonic conductivity at high temperatures.

We consider the following correlator in the conductivity relation (12) which characterizes the hopping transport

$$
K_{\text {hop }}=\langle\tilde{J}(z) \tilde{J}(0)\rangle_{h o p}=K_{\text {hop }}^{T}+K_{\text {hop }}^{R}+K_{\text {hop }}^{T R}
$$

where $z=t-i \hbar \lambda$ and

$$
\begin{aligned}
K_{h o p}^{T}= & \frac{e^{2} \Omega_{T}^{2}}{\hbar^{2}} R_{a b}^{2} \sum_{l l^{\prime}}\left(\left\langle\tilde{\Phi}_{T}^{l}(z) c_{l a}^{+}(z) c_{l b}(z) \tilde{\Phi}_{T}^{l^{\prime *}} c_{l^{\prime} b}^{+} c_{l^{\prime} a}\right\rangle+\left\langle\tilde{\Phi}_{T}^{l *}(z) c_{l b}^{+}(z) c_{l a}(z) \tilde{\Phi}_{T}^{l^{\prime}} c_{l^{\prime} a}^{+} c_{l^{\prime} b}\right\rangle\right), \\
K_{h o p}^{R}= & \frac{e^{2} \Omega_{R}^{2}}{\hbar^{2}} R_{r}^{2} \sum_{l l^{\prime}}\left(\left\langle\tilde{\Phi}_{R}^{l}(z) c_{l-1, b}^{+}(z) c_{l a}(z) \tilde{\Phi}_{R}^{l^{\prime *}} c_{l^{\prime} a}^{+} c_{l^{\prime}-1, b}\right\rangle+\right. \\
& \left.\left\langle\tilde{\Phi}_{R}^{l *}(z) c_{l a}^{+}(z) c_{l-1, b}(z) \tilde{\Phi}_{R}^{l^{\prime}} c_{l^{\prime}-1, b}^{+} c_{l^{\prime} a}\right\rangle\right),
\end{aligned}
$$




$$
\begin{aligned}
K_{h o p}^{T R}= & -\frac{e^{2} \Omega_{0} \Omega_{R}}{\hbar^{2}} R_{a b} R_{r} \sum_{l l^{\prime}}\left(\left\langle\tilde{\Phi}_{T}^{l}(z) c_{l a}^{+}(z) c_{l b}(z) \tilde{\Phi}_{R}^{l^{\prime *}} c_{l^{\prime}-1, b}^{+} c_{l^{\prime} a}\right\rangle+\right. \\
& \left\langle\tilde{\Phi}_{T}^{l *}(z) c_{l b}^{+}(z) c_{l a}(z) \tilde{\Phi}_{R}^{l^{\prime}} c_{l^{\prime} a}^{+} c_{l^{\prime}-1, b}\right\rangle+\left\langle\tilde{\Phi}_{R}^{l *}(z) c_{l-1, b}^{+}(z) c_{l a}(z) \tilde{\Phi}_{T}^{l^{\prime}} c_{l^{\prime} a}^{+} c_{l^{\prime} b}\right\rangle+ \\
& \left.\left\langle\tilde{\Phi}_{R}^{l}(z) c_{l a}^{+}(z) c_{l-1, b}(z) \tilde{\Phi}_{T}^{l^{\prime} *} c_{l^{\prime} b}^{+} c_{l^{\prime} a}\right\rangle\right)
\end{aligned}
$$

Here the operators $\tilde{\Phi}_{\alpha}^{l}=\Phi_{\alpha}^{l}-\left\langle\Phi_{\alpha}^{l}\right\rangle(\alpha=T, R)$ correspond to the phonon scattering processes which accompany the proton transfer. We perform evaluation of (18) using the Hamiltonian with averaged values of the transfer integrals $\tilde{\Omega}_{\alpha}$. This allows us to decouple the protonphonon correlation functions

$$
\left\langle\tilde{\Phi}_{\alpha}^{m}(z) c_{l \nu}^{+}(z) c_{l^{\prime} \nu^{\prime}}(z) \tilde{\Phi}_{\alpha_{1}}^{m_{1}} c_{l_{1}, \nu_{1}}^{+} c_{l_{1}^{\prime} \nu_{1}^{\prime}}\right\rangle \rightarrow\left\langle\tilde{\Phi}_{\alpha}^{m}(z) \tilde{\Phi}_{\alpha_{1}}^{m_{1}}\right\rangle\left\langle c_{l \nu}^{+}(z) c_{l^{\prime} \nu^{\prime}}(z) c_{l_{1}, \nu_{1}}^{+} c_{l_{1}^{\prime} \nu_{1}^{\prime}}\right\rangle
$$

into proton and phonon parts which can be evaluated separately. The processes that we are interested in are going on at considerably high temperatures and in the strong protonphonon coupling regime (we will see below that the strong polaron effect actually occurs in these systems). This allows us to assume

$$
\sum_{q} \frac{\left|\tau_{l}(q)\right|^{2}}{(\hbar \omega(q))^{2} \operatorname{sh} \beta \hbar \omega(q) / 2}>>1 .
$$

We take a dispersionless approximation for the phonon frequency: $\omega(q)=\omega_{0}$. In this case $E_{0}=(\hbar g)^{2} / 2 M\left(\hbar \omega_{0}\right)^{2}$ and evaluation of the phonon correlation functions yields

$$
\begin{aligned}
& \psi_{T T}=\left\langle\tilde{\Phi}_{T}^{l}(z) \tilde{\Phi}_{T}^{l^{\prime} *}\right\rangle=\left\langle\tilde{\Phi}_{T}^{l *}(z) \tilde{\Phi}_{T}^{l^{\prime}}\right\rangle=\mathrm{e}^{-\beta E_{0} / 2} \mathrm{e}^{-(z+i \hbar \beta / 2)^{2} / 4 \tilde{\tau}_{T}^{2}} \delta_{l l^{\prime}}+ \\
& \mathrm{e}^{-\beta E_{0} / 4} \mathrm{e}^{-\frac{E_{0}}{\hbar \omega_{0}} \operatorname{cth} \beta \hbar \omega_{0} / 2} \mathrm{e}^{-(z+i \hbar \beta / 2)^{2} / 4 \tilde{\tau}_{T}^{\prime 2}} \delta_{l, l^{\prime} \pm 1}, \\
& \psi_{R R}=\left\langle\tilde{\Phi}_{R}^{l}(z) \tilde{\Phi}_{R}^{l^{\prime} *}\right\rangle=\left\langle\tilde{\Phi}_{R}^{l *}(z) \tilde{\Phi}_{R}^{l^{\prime}}\right\rangle=\mathrm{e}^{-\beta E_{0}} \mathrm{e}^{-(z+i \hbar \beta / 2)^{2} / 4 \tilde{\tau}_{R}^{2}} \delta_{l l^{\prime}}, \\
& \psi_{T R}=\left\langle\tilde{\Phi}_{T}^{l}(z) \tilde{\Phi}_{R}^{l^{\prime} *}\right\rangle=\left\langle\tilde{\Phi}_{T}^{l *}(z) \tilde{\Phi}_{R}^{l^{\prime}}\right\rangle=\left\langle\tilde{\Phi}_{R}^{l}(z) \tilde{\Phi}_{T}^{l^{\prime} *}\right\rangle=\left\langle\tilde{\Phi}_{R}^{l *}(z) \tilde{\Phi}_{T}^{l^{\prime}}\right\rangle=\mathrm{e}^{-\beta E_{0} / 2} \times \\
& \mathrm{e}^{-\frac{E_{0}}{\hbar \omega_{0}} \operatorname{cth} \beta \hbar \omega_{0} / 2} \mathrm{e}^{-(z+i \hbar \beta / 2)^{2} / 4 \tilde{\tau}_{T}^{2}}\left(\delta_{l l^{\prime}}+\delta_{l, l^{\prime} \pm 1}\right)
\end{aligned}
$$

The parameters introduced in (21)

$$
\tilde{\tau}_{T}^{2}=\frac{\beta \hbar^{2}}{8 E_{0}}, \quad \tilde{\tau}_{R}^{2}=\frac{\beta \hbar^{2}}{16 E_{0}}, \quad \tilde{\tau}_{T}^{\prime 2}=\frac{\beta \hbar^{2}}{4 E_{0}}
$$


characterize the average hopping time lengths between two localized positions in the chain (the hopping time within H-bond, the interbond reorientational hopping time and the time for pair polaron transfer within the neighbouring bonds respectively).

Next we consider the proton part of the correlation functions. We introduce the $k$ representation for the Fermi operators

$$
c_{l \nu}=\frac{1}{\sqrt{N}} \sum_{k} c_{k \nu} \mathrm{e}^{i k l d}
$$

and further perform the following unitary transformation

$$
\begin{aligned}
c_{k a} & =\frac{1}{d_{k}}\left(\left[\tilde{t}_{k}-i\left|\tilde{t}_{k}\right|\right] \tilde{c}_{k a}-\left[\left|\tilde{t}_{k}\right|+i \tilde{t}_{k}\right] \tilde{c}_{k b}\right), \\
c_{k b} & =\frac{1}{d_{k}}\left(\left[\left|\tilde{t}_{k}\right|-i \tilde{t}_{k}^{*}\right] \tilde{c}_{k a}+\left[\tilde{t}_{k}^{*}+i\left|\tilde{t}_{k}\right|\right] \tilde{c}_{k b}\right)
\end{aligned}
$$

where $d_{k}=2 \sqrt{\left|\tilde{t}_{k}\right|} \sqrt{\left|\tilde{t}_{k}\right|-\Im \tilde{t}_{k}}$. Then the polaron Hamiltonian can be reduced to the diagonal form $\sum_{k \nu} \varepsilon_{k \nu} \tilde{n}_{k \nu}\left(\varepsilon_{k \nu}=-\tilde{\mu} \pm\left|\tilde{t}_{k}\right|\right)$ and the correlation functions $\left\langle\tilde{c}_{k \nu}^{+}(z) \tilde{c}_{k^{\prime} \nu^{\prime}}(z) \tilde{c}_{k_{1} \nu_{1}}^{+} \tilde{c}_{k_{1}^{\prime} \nu_{1}^{\prime}}\right\rangle$ can be evaluated using the Wick's theorem (in this case we use the Schrödinger representation for the polaron operators $\left.\tilde{c}_{k \nu}(z)=\mathrm{e}^{-i \varepsilon_{k \nu} z / \hbar} \tilde{c}_{k \nu}\right)$.

We use procedure proposed in 15 with the deformation of the integration contour in the complex plane for the integration of expressions (18) over $t$ and $\lambda$. Let us present the resulting expression for the real part of the conductivity that will be analyzed afterwards

$$
\begin{aligned}
& \sigma_{\text {hop }}(\omega, T)=\sigma_{\text {hop }}^{0}(\omega, T)+\sum_{\nu \nu^{\prime}} \sigma_{\text {hop }}^{\nu, \nu^{\prime}}(\omega, T), \\
& \sigma_{\text {hop }}^{0}(\omega, T)=\frac{2 e^{2} \sqrt{\pi}}{d N^{2} \hbar^{2}} \frac{\operatorname{sh} \beta \hbar \omega / 2}{\hbar \omega / 2} \sum_{k k^{\prime}}\left\{\left(C_{T} \mathrm{e}^{-\tilde{\tau}_{T}^{2} \omega^{2}}+C_{T}^{\prime} \mathrm{e}^{-\tilde{\tau}_{T}^{\prime 2} \omega^{2}}\right) \Re\left(\tilde{t}_{k}^{*} \tilde{t}_{k^{\prime}}\right)+\right. \\
& \left.C_{R} \mathrm{e}^{-\tilde{\tau}_{R}^{2} \omega^{2}} \Re\left(\tilde{t}_{k}^{*} \tilde{t}_{k^{\prime}} \mathrm{e}^{-i\left(k-k^{\prime}\right) d}\right)+C_{T R} \mathrm{e}^{-\tilde{\tau}_{T}^{2} \omega^{2}} \Re\left(\tilde{t}_{k}^{*} \tilde{t}_{k^{\prime}}\left[\mathrm{e}^{i k^{\prime} d}+\mathrm{e}^{-i k d}\right]\right)\right\} \frac{\left(\left\langle\tilde{n}_{k a}\right\rangle-\left\langle\tilde{n}_{k b}\right\rangle\right)\left(\left\langle\tilde{n}_{k^{\prime} a}\right\rangle-\left\langle\tilde{n}_{k^{\prime} b}\right\rangle\right)}{\left|\tilde{t}_{k}\right|\left|\tilde{t}_{k^{\prime}}\right|} \\
& \sigma_{\text {hop }}^{\nu, \nu^{\prime}}(\omega, T)=\frac{2 e^{2} \sqrt{\pi}}{d N^{2} \hbar^{2}} \frac{\operatorname{sh} \beta \hbar \omega / 2}{\hbar \omega / 2} \sum_{k k^{\prime}}\left\{C_{T} \mathrm{e}^{-\tilde{\tau}_{T}^{2} \tilde{\omega}^{2}}+C_{T}^{\prime} \mathrm{e}^{-\tilde{\tau}_{T}^{\prime 2} \tilde{\omega}^{2}} \cos \left(k-k^{\prime}\right) d+\right. \\
& \left.C_{R} \mathrm{e}^{-\tilde{\tau}_{R}^{2} \tilde{\omega}^{2}}+C_{T R} \mathrm{e}^{-\tilde{\tau}_{T}^{2} \tilde{\omega}^{2}}\left(\cos k d+\cos k^{\prime} d\right)\right\} \mathrm{e}^{\beta\left(\varepsilon_{k \nu}-\varepsilon_{k^{\prime} \nu^{\prime}}\right) / 2}\left\langle\tilde{n}_{k \nu}\right\rangle\left(1-\left\langle\tilde{n}_{k^{\prime} \nu^{\prime}}\right\rangle\right) . \\
& C_{T}=\Omega_{T}^{2} R_{a b}^{2} \tilde{\tau}_{T} \mathrm{e}^{-\beta E_{0} / 2}, \quad C_{T}^{\prime}=2 \Omega_{T}^{2} R_{a b}^{2} \tilde{\tau}_{T}^{\prime} \mathrm{e}^{-\beta E_{0} / 4} \mathrm{e}^{-\frac{E_{0}}{\hbar \omega_{0}} \operatorname{cth} \beta \hbar \omega_{0} / 2}, \\
& C_{R}=\Omega_{R}^{2} R_{r}^{2} \tilde{\tau}_{R} \mathrm{e}^{-\beta E_{0}}, \quad C_{T R}=-2 \Omega_{T} \Omega_{R} R_{a b} R_{r} \tilde{\tau}_{T} \mathrm{e}^{-\beta E_{0} / 2} \mathrm{e}^{-\frac{E_{0}}{\hbar \omega_{0}} \operatorname{cth} \beta \hbar \omega_{0} / 2} .
\end{aligned}
$$


The first term in (23) appears in the result of pairing of the operators $\tilde{c}_{k \nu}^{+}$with $\tilde{c}_{k^{\prime} \nu^{\prime}}$ in the proton correlation functions with equal values of time argument, whereas another terms $\sigma_{h o p}^{\nu, \nu^{\prime}}$ arise due to the pairing of such operators with different time arguments.

Let us discuss first the frequency dependence of the hopping conductivity. From expression (23) it follows that $\sigma_{\text {hop }}$ has several peaks which point to the important contribution of various elementary dynamical processes existing in the system. It is possible to estimate analytically in the limit $\beta \hbar \omega>>1$ (in the high frequency range or at lower temperatures) the characteristic frequency values at which the conductivity maxima exist. The first maximum at $\omega_{1} \sim \frac{\beta \hbar}{4 \tilde{\tau}_{T}^{\prime 2}}$ corresponds to the pair phonon-activated hopping within the neighbouring H-bonds. Another maximum at $\omega_{2} \sim \frac{\beta \hbar}{4 \tilde{\tau}_{T}^{2}}$ arises due to the intrabond polaron dynamics, whereas the peak at $\omega_{3} \sim \frac{\beta \hbar}{4 \tilde{\tau}_{R}^{2}}$ is associated with the reorientational hopping of the protonic polaron between bonds. The frequency dependencies of the real and imagine parts of the hopping conductivity are shown in Figs. 2, 3 for different temperatures and values of polaron binding energy $E_{0}$. We observe three distinct peaks at sufficiently low temperatures, which broaden significantly when temperature increase. Finally, at a considerably high temperatures the behavior $\sigma_{\text {hop }}(\omega)$ has the Drude-type character with maximum at $\omega=0$ and smoothing decrease with increasing frequency.

The two-stage transport mechanism manifests itself in the two peaks at $\omega=\omega_{2}$ and $\omega=\omega_{3}$ observed in the frequency dependence of the optical conductivity which shift to the high-frequency region when the polaron binding energy $E_{0}$ increases (see Fig. 3). With the increase of $E_{0}$ the conductivity lowers and the maxima broaden due to the stronger localization of protonic polaron in one of the potential well of the hydrogen bond. We note that the values of the hopping integrals $\tilde{\Omega}_{T}$ and $\tilde{\Omega}_{R}$ practically do not influence on the positioning of these maxima but affect strongly the redistribution of the maxima heights.

Figure 4 shows the comparison of theoretical conductivity obtained for lithium hydrazinium sulphate $\mathrm{Li}\left(\mathrm{N}_{2} \mathrm{H}_{5}\right) \mathrm{SO}_{4}$ (LHS) using (23) with experimentally measured values 12 at the frequency $500 \mathrm{KHz}$. This compound consists of the chains of hydrogen bonded hydrazinium ions running along the [001] direction and can be considered as the example of 
quasi-one-dimensional protonic conductor 13 . In this case we obtain $\hbar \omega_{0}=800 \mathrm{~cm}^{-1}$ and $E_{0}=0.98 \mathrm{eV}$. According to our results, the dominant contribution to the conductivity in this frequency range arises from the intrabond hopping with $\Omega_{T}=0.04 \mathrm{eV}$. The relation $E_{0}>2 \Omega_{T}$ follows immediately from the values of our parameters and is valid for the small-polaron regimen.

Let us analyze the theoretical hopping conductivity in a wide temperature interval for the various system parameters. Since the proton is localized in the chain due to the strong polaronic effect, we expect intuitively that change in an external control parameters may have a determining influence on the proton state and lead to its delocalization. In particular, it is of prime importance to analyze in more details the influence of temperature. On the one hand, it is well known that the effect of temperature is dramatic in the systems which undergo the transitions to superprotonic phases, which accompanied by significantly increase of the protonic conductivity. In this case the proton state changes especially drastically: in the lowtemperature ordered phases the protons are localized in well-defined positions of the lattice and can migrate with sufficiently high activation energy $(\sim 1 \mathrm{eV})$ due to the strong coupling with displacements of ionic groups participating in hydrogen bond formation, whereas in the superionic phases the transport phenomenon is connected with dynamically disorder of the hydrogen bond network, resulting in an increase of the number of possible positions for protons which can hop between them with much lower activation energy $(\sim 0.1 \mathrm{eV})$. On the other hand, the influence of temperature on the localized state of protonic polaron can be especially important in a variety of protonic conductors, both in inorganic systems and biological membranes. Because of this, we consider the temperature dependence of the hopping conductivity at the given frequency of external field.

The real part of the $\sigma_{\text {hop }}$ as a function of temperature has peculiarities which arise due to delocalization effects. We consider several characteristic frequency regions: $\omega \sim \omega_{2}$ (here the intrabond hopping contributes mainly to the total conductivity), $\omega \sim \omega_{3}$ (when the reorientational interbond hopping dominates) and $\omega=0$ (static de conductivity). The effect of two-stage transport process shows itself most clearly in the high-frequency region. The 
first case is shown in Fig. 5. The two maxima are observed which shift slightly towards the higher temperatures when $E_{0}$ increases. It is easy to understand the origin of this nonmonotonic behavior of $\sigma_{\text {hop }}$ from Fig. 6, where the temperature dependence of $\sigma_{\text {hop }}$ is compared with two extreme cases that one of the transport component (interbond or intrabond) is eliminated. Now we can conclude immediately that the first sharp increase of $\sigma_{\text {hop }}$ with further rapid lowering originates from the intrabond hopping, whereas the second smoother maximum on higher temperature interval corresponds to the interbond contribution. The raising of temperature suppresses the polaron localization effect: the thermal proton dynamics leads to the detrapping of protonic polaron which accompanied by drastically increase of $\sigma_{\text {hop }}$. However, with the further temperature increasing the conductivity lowers again due to the multiphonon scattering processes suggesting that the system undergoes a transition to the metallic-type state. Therefore, we can conclude that in our system the possible transition from the insulator state has a two-step character: first the protonic polaron is delocalized within the H-bond, and only with further temperature increase it becomes delocalized between bonds. The situation changes alternatingly in the vicinity of $\omega \sim \omega_{3}$ (see Figs. 7 and 8). We see that in this case first the tendency for interbond delocalization occurs, whereas the intrabond contribution dominates at higher temperatures. The last case $\sigma_{\text {hop }}(\omega=0)$ is shown in Fig. 9. for different relations between $\Omega_{T}$ and $\Omega_{R}$. We see the single maximum which corresponded to the transition to metallic-type state appeared due to the dominant contribution of reorientations (when $\Omega_{R}>\Omega_{T}$, Fig. 9(a)) or intrabond hopping (when $\Omega_{T}>\Omega_{R}$, Fig. 9(b)). In the case $\Omega_{T} \sim \Omega_{R}$ (Fig. 9(c)) the both parts contribute practically equally to the transition occurrence. It is worth noting that despite of the single smooth peak, we can also distinguish two steps in the dc conductivity behavior which becomes clear after comparison of our curves with the two extreme cases $\left(\Omega_{R}=0\right.$ and $\left.\Omega_{T}=0\right)$. We can conclude that the protonic polarons become delocalized between bonds at lower temperatures, and the intrabond delocalization proceeds on further heating. This effect can be explained by the fact that the reorientational hopping has higher activation energy than the interbond motion (see expressions (23) for the conductivity) and thus the 
first type of hopping has the tendency to damp at lower temperatures due to the multiphonon scattering than the second type of hopping. Thus our model allows us a possibility to analyze thoroughly the effect of either of two transport stages on the total conductivity picture that can be verified by experimental measurements.

\section{CONCLUSIONS}

In the present work the protonic conductivity of the quantum quasi-one-dimensional hydrogen-bonded chain has been studied in the framework of the two-stage orientationaltunneling model. The interaction of protons with the optical stretching mode is considered. The expression for the conductivity coefficient as a function of temperature and frequency is obtained using the Kubo formula for various values of hopping parameters and polaron binding energy. The evaluated hopping conductivity is in good agreement with the experimentally measured values for LHS system. This allows us to conclude that the strong proton-phonon coupling regime exists in this type of systems. The analysis of theoretically

obtained temperature dependencies of $\sigma_{h o p}$ in wide temperature interval revealed that the transition from the insulator state (increase of $\sigma$ with increase of $\mathrm{T}$ ) to the metal-type state (decrease of $\sigma$ with increase of $\mathrm{T}$ ) occurs in system on heating. The specific two-stage Grotthuss transport mechanism manifests itself in the two-step character of the transition as well as in the two distinct peaks of dynamical conductivity. The obtained theoretical results allow us to believe that the proposed theory should also apply to other hydrogen bonded systems and to investigate the consequences of assumptions of the Grotthuss mechanism for proton migration in hydrogen bonded compounds.

\section{ACKNOWLEDGEMENTS}

This work is partially supported by INTAS Grant No. 95-0133. 


\section{REFERENCES}

${ }^{1}$ D. Hadži, J. Mol. Struct. 177, 1 (1988).

${ }^{2}$ A. Kawada, A. R. McGhie and M. M. Labes, J. Chem. Phys. 52, 3121 (1970).

${ }^{3}$ M.-S. Chen, L. Onsager, J. Bonner and J. Nagle, J. Chem. Phys. 60, 405 (1974).

${ }^{4}$ A. V. Belushkin, C. J. Carlile and L. A. Shuvalov, Ferroelectrics 167, 21 (1995).

${ }^{5}$ P. Zetterström, A. V. Belushkin, R. L. McGreevy and L. A. Shuvalov, Solid State Ionics 167, 21 (1999).

${ }^{6}$ A. Pietraszko, B. Hilczer and A. Pawlowski, Solid State Ion. 119(1-4), 281 (1999)

${ }^{7}$ A. Pietraszko, K. Łukaszewicz and M.A. Augustyniak, Acta Cryst. C 48, 2069 (1992);

K. Łukaszewicz, A. Pietraszko and M.A. Augustyniak, ibid. 49, 430 (1993); A. Pietraszko and K. Łukaszewicz, Bull. Polish Acad. Sci. 41, 157 (1993).

${ }^{8}$ N. Pavlenko, J.Phys.: Condens. Matter 11, 5099 (1999).

${ }^{9}$ S. V. Kravchenko, G. V. Kravchenko, J. E. Furneaux, V. N. Pudalov and M. D’Iorio, Phys. Rev. B 508039 (1994).

${ }^{10}$ I. V. Stasyuk, O. L. Ivankiv and N. Pavlenko J. Phys. Stud. 1, 418 (1997).

${ }^{11}$ Yu. A. Firsov. Polarons, (Nauka, Moscow, 1975, in Russian).

${ }^{12}$ F. E. Salman, B. Hilczer and Cz. Pawlaczyk, Japanese J. Appl. Phys. 24, 668 (1985).

${ }^{13}$ V. M. Padmanabhan and R. Balasubramanian, Acta Cryst. 22, 532 (1967).

${ }^{14}$ Kubo R 1957 J. Phys. Soc. Jpn. 12, 570

${ }^{15}$ H. G. Reik and D. Heese, J.Phys. Chem. Solids 28581 (1967). 


\section{FIGURE CAPTIONS}

Figure 1. (a) Zig-zag hydrogen-bonded chain, arrows indicate the possible path of proton migration along the chain. (b) Simplified model chains, the in-phase displacements of ionic groups identified by the dashed arrows.

Figure 2. Frequency dependencies of the hopping conductivity $\tilde{\sigma}_{h o p}(\tilde{\omega})=\sigma_{h o p} / c_{0}$ $\left(c_{0}=2 \frac{e^{2}}{d \hbar} r_{0}^{2}, \tilde{\omega}=\omega / \omega_{0}\right)$ for $\tilde{E}_{0}=E_{0} / \hbar \omega_{0}=2$ and different temperatures $\tau=k T / \hbar \omega_{0} ;$ $R_{a b} / r_{0}=1.3, R_{r} / r_{0}=1.6 . \omega_{T}=\tilde{\Omega}_{T} / \hbar \omega_{0}=0.8$ and $\omega_{R}=\tilde{\Omega}_{R} / \hbar \omega_{0}=0.5$ are reduced hopping amplitudes. The real and imagine parts are indicated by bold and thin curves respectively.

Figure 3. Frequency dependencies of $\Re \tilde{\sigma}_{h o p}$ at $\tau=0.25$ for different values of $\tilde{E}_{0}$ and hopping amplitudes.

Figure 4. Comparison of the temperature dependencies of the protonic conductivity, measured for the crystal LHS and evaluated using (23).

Figure 5. Temperature dependencies of $\Re \tilde{\sigma}_{h o p}$ for different values of $E_{0}$ at $\tilde{\omega}=5.5, \omega_{T}=0.2$ and $\omega_{R}=0.6$.

Figure 6. Comparison of $\Re \tilde{\sigma}_{h o p}(T)$ for $\tilde{E}_{0}=2.5, \tilde{\omega}=5.5, \omega_{T}=0.2$ and $\omega_{R}=0.6$ with two extreme cases when the intrabond or interbond contribution is eliminated.

Figure 7. Temperature dependencies of $\Re \tilde{\sigma}_{h o p}$ for different values of $E_{0}$ at $\tilde{\omega}=8.7, \omega_{T}=0.6$ and $\omega_{R}=0.3$.

Figure 8. Comparison of $\Re \tilde{\sigma}_{h o p}(T)$ for $\tilde{E}_{0}=2.3, \tilde{\omega}=8.7, \omega_{T}=0.6$ and $\omega_{R}=0.3$ with two extreme cases when the intrabond or interbond contribution is eliminated.

Figure 9. Temperature dependencies of dc hopping conductivity for different values of $\omega_{T}$ and $\omega_{R}$ at $\tilde{E}_{0}=2.5$. 


\section{FIGURES}

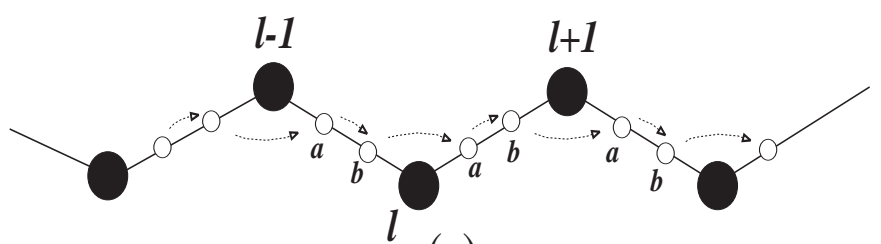

(a)
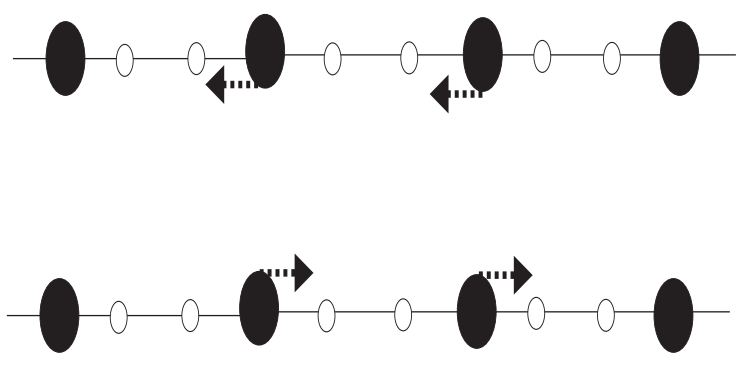

(b)

FIG. 1.

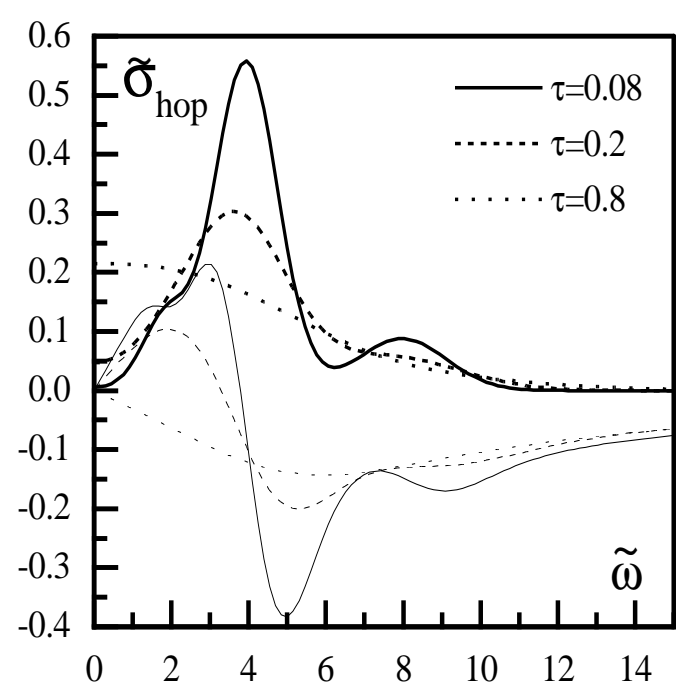

FIG. 2. 


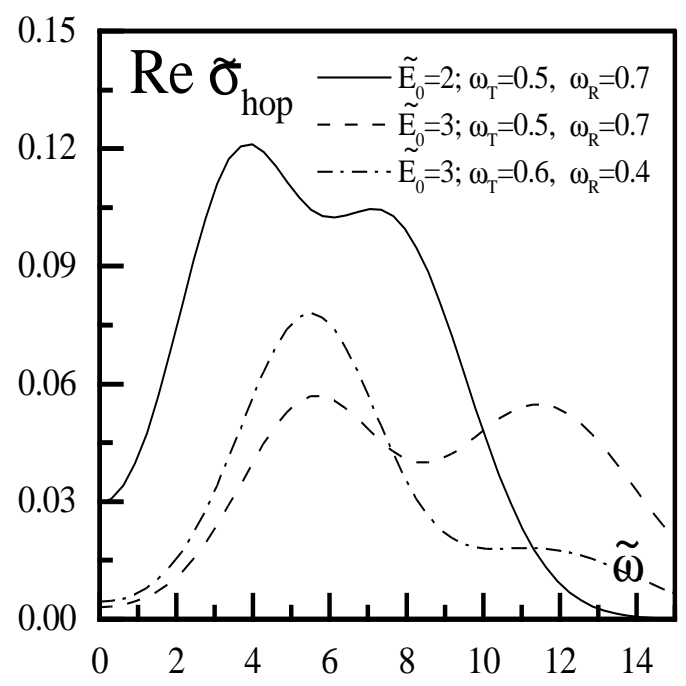

FIG. 3.

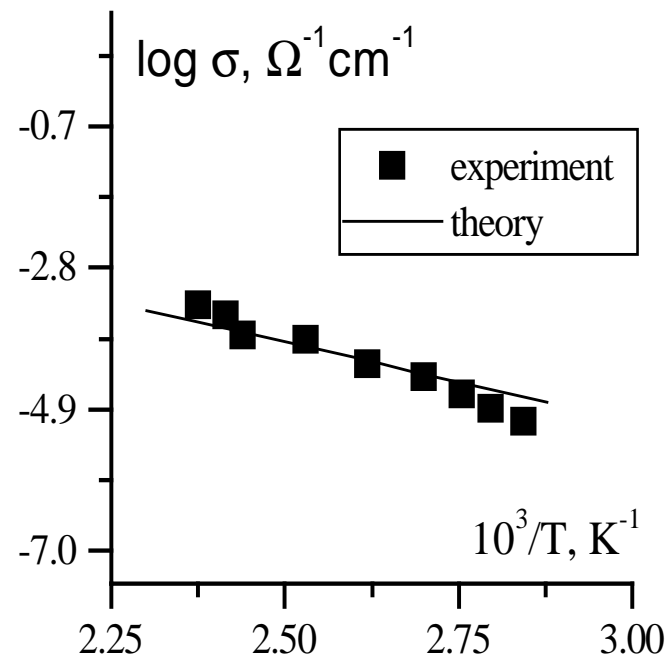

FIG. 4. 


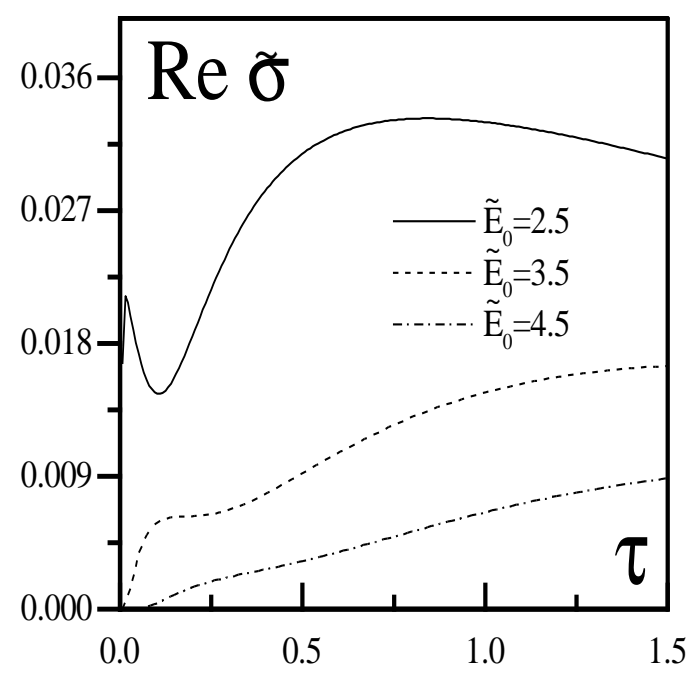

FIG. 5.

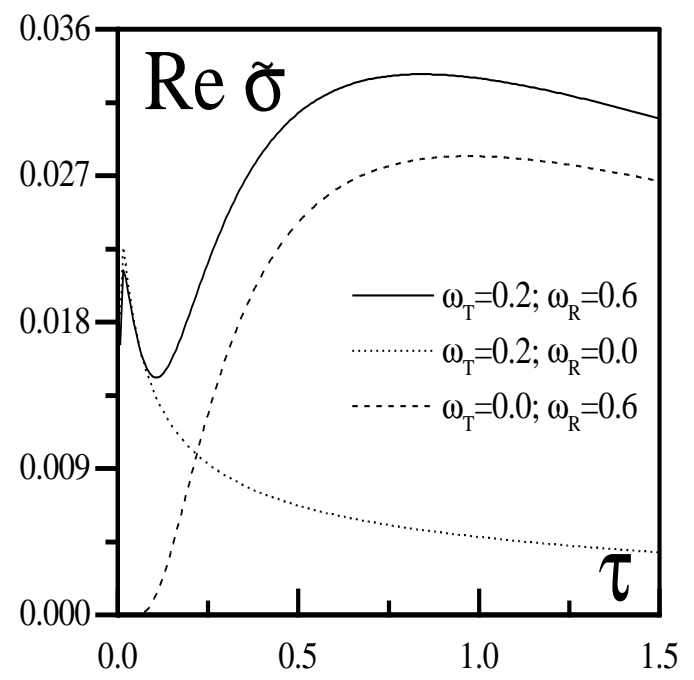

FIG. 6. 


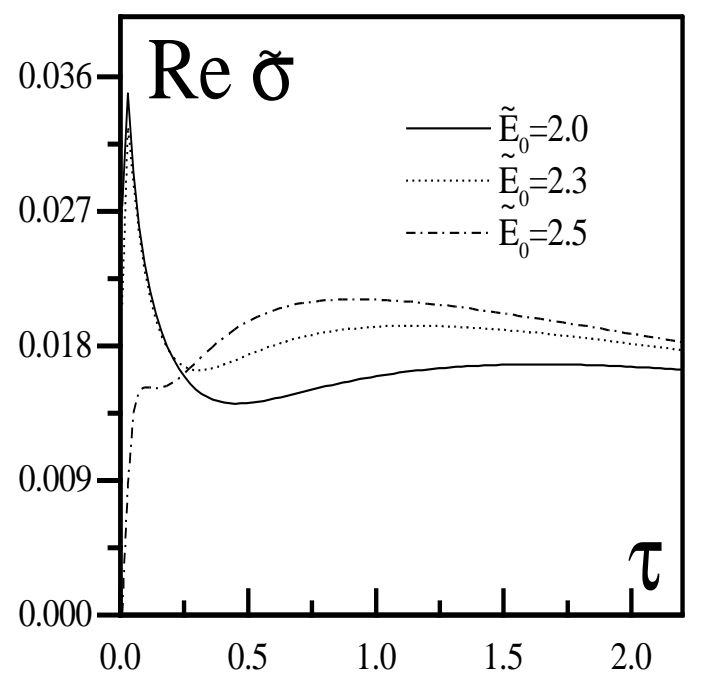

FIG. 7.

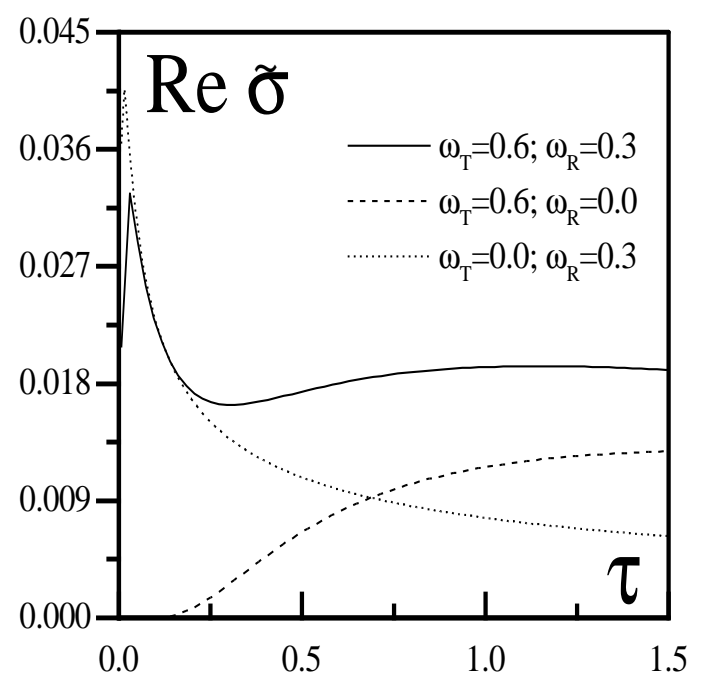

FIG. 8. 

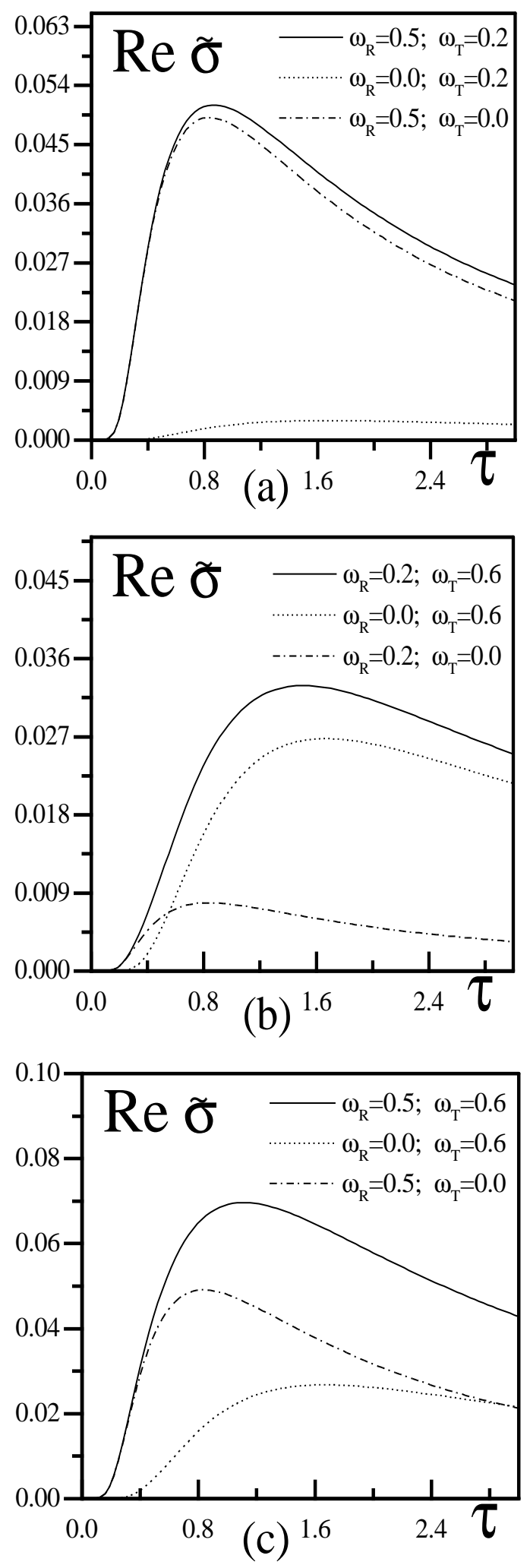

FIG. 9. 